\title{
The X-ray Evolving Universe Spectroscopy Mission (XEUS)
}

M. Bavdaz', J.A.M. Bleeker ${ }^{2}$, G. Hasinger ${ }^{3}$, H. Inoue ${ }^{4}$, G. Palumbo ${ }^{5}$, A. Peacock ${ }^{1}$, A. Parmar ${ }^{1}$, M.J. Turner ${ }^{6}$, J. Truemper ${ }^{7}$, J. Schiemann ${ }^{8}$

'Space Science Department of ESA, ESTEC, Noordwijk, The Netherlands

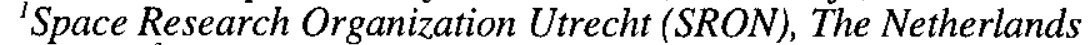

${ }^{3}$ Astrophysikalisches Institut Potsdam, Germany

${ }^{4}$ Institute of Space and Astronautical Science (ISAS), Japan

${ }^{5}$ Dipartmento dipartimento di Astronomia, Universita degli Studi di Bologna, Italy

${ }^{6}$ Department of Physics and Astronomy, Leicester University, United Kingdom

${ }^{7}$ Max Planck Institut für Physik und Astrophysik, Garching, Germany

${ }^{8}$ Directorate of Manned Spaceflight and Microgravity ESA, ESTEC, Noordwijk, The Netherlands

ESLAB 1998/051/SA

To be published in SPIE Proceedings V3766

Presented at the SPIE International Symposium on Optical Science, Engineering and Instrumentation, 18-23 July 1999, Denver, USA 


\title{
The X-ray Evolving Universe Spectroscopy Mission (XEUS)
}

\author{
M. Bavdaz ${ }^{* 1}$, J.A.M. Bleeker ${ }^{2}$, G. Hasinger ${ }^{3}$, H. Inoue ${ }^{4}$, G. Palumbo ${ }^{5}$, A.Peacock ${ }^{1}$, A. Parmar ${ }^{1}$, \\ M.J. Turner ${ }^{6}$, J. Truemper $^{7}$, J. Schiemann ${ }^{8}$ \\ ${ }^{1}$ Space Science Department of ESA, ESTEC, Noordwijk, Netherlands \\ ${ }^{2}$ Space Research Organization Utrecht, Netherlands, \\ ${ }^{3}$ Astrophysikalisches Institut Potsdam, Germany, \\ ${ }^{4}$ Institute of Space and Astronautical Science (ISAS), Japan, \\ ${ }^{5}$ Dipartimento di Astronomia, Universita degli Studi di Bologne, Italy, \\ ${ }^{6}$ Dept. Physics and Astronomy,Leicester University,United Kingdom, \\ ${ }^{7}$ Max Planck Institut Physik und Astrophysik, Garching, Germany, \\ ${ }^{8}$ Directorate Of Manned Spaceflight and Microgravity ESA, ESTEC, Noordwijk, Netherlands
}

\begin{abstract}

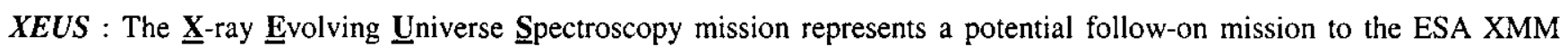
cornerstone currently nearing completion. XEUS represents the next logical step forward in X-ray astrophysics after the current set of missions have been launched and completed their operational lives. The development and ultimate success relies heavily on the capability of the International Space Station (ISS). In this paper we describe the key characteristics of the mission including the requirements placed specifically on the ISS and discuss the significant advances in high energy astrophysics expected from such an observatory.
\end{abstract}

The aim of XEUS is to study the astrophysics of some of the most distant and hence youngest known discrete objects in the universe. The specific scientific issues, which XEUS aims to address, can be summarized as follows:

- To measure the X-ray spectra of objects with a redshift $\mathrm{z}>4$ at flux levels about $<10^{-17} \mathrm{erg} \mathrm{cm}^{-2} \mathrm{~s}^{-1}$. This is at least a 100 times fainter than XMM.

- Where possible to determine from the X-ray spectral lines the redshift and thus the age of these objects

- To thereby establish the evolution in the distribution of matter in the early universe

To achieve these demanding aims a large $\mathrm{X}$-ray telescope is required. The basic features of this telescope are:

- An X-ray mirror with an effective collecting area at $1 \mathrm{keV}$ of $30 \mathrm{~m}^{2}$.

- A spatial resolution of the mirror needs to be at least 5" (HEW) so as to avoid source confusion at above mentioned faint source levels.

- A field of view of at least $5^{\prime}$ is required so as to ensure that a significantly large population of high redshift $\mathrm{x}$-ray sources can be observed in a single pointing.

- Energy bandwith required is 0.05 to $30 \mathrm{keV}$, with $3 \mathrm{~m}^{2}$ at $8 \mathrm{keV}$.

- An energy resolution of 1-10 eV is required to perform detailed plasma diagnostics on sources, such as distant galaxy clusters

These are some of the most demanding requirements yet placed on any X-ray astrophysics mission and will require major technological development at all levels - optics, detectors and spacecraft.

\section{Key Words : X-ray Astrophysics, Future missions, Evolving universe}

\footnotetext{
"Correspondence: E-mail: mbavdaz@estec.esa.nl; Telephone: +31 71565 4933; Fax: +31 71 565 4690; ESA/ESTEC, SCISA, P.O.Box 299, NL-2200AG Noordwijk, The Netherlands
} 


\section{INTRODUCTION}

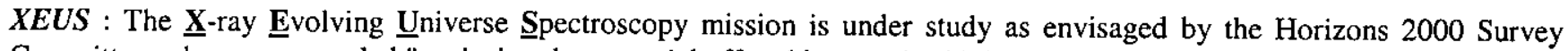
Committee, who recommended "analysing the potential offered by a major high energy astrophysics facility within the Space Station (ISS) Utilization Programme". The original mission concept has arisen through extensive discussions by the European Scientific Community, particularly at the Workshop held at Leicester University UK in July 1996. At this international workshop the foundations for the "Next Generation of X-ray Observatories" was laid. With XMM due for launch in early 2000, with a mission duration of 5-10 years, it is not too early to consider the post XMM era. At the turn of the century two great X-ray observatories will have embarked on their astrophysics programs - XMM and NASA's Chandra and thus the requirements of XEUS must take account of the key thrusts and potential discoveries from both these powerful missions.

The XEUS mission aims to do this by placing a permanent X-ray observatory in space, that provides a telescope aperture equivalent to the largest ground based optical telescopes - essentially the equivalent of the VLT for X-ray astronomy in space. By making full use of the facilities available at the ISS in the next century and by ensuring in the XEUS design a significant potential for growth and evolution, the overall mission lifetime of XEUS could be well over a quarter of a century. The power of this observatory will be such that for the first time detailed imaging spectroscopy studies will be undertaken in high energy astrophysics of objects associated with the evolution of the early universe.

\section{THE SCIENTIFIC RATIONALE FOR XEUS}

The astrophysics of the 21 st century will largely concentrate on the study of the high-redshift universe. This will allow fundamental questions to be addressed,such as:

- How did the first galaxies form?

- What is the history of the large-scale structure visible today?

- How and when were the elements created?

- What were the first discrete objects?

- What is the nature of dark matter?

ESA's Plank mission will measure the cosmological parameters to high precision while NGST, FIRST and ALMA will probe the time when the first stars emerged and provide information about the formation and evolution of galaxies. A powerful Xray mission such as XEUS will provide the additional observations necessary to study the hot universe at high-redshift with comparable sensitivity as these other facilities. X-ray observations can provide information about the creation of the first black holes and study the strong gravitational field in the immediate vicinity of these objects. X-rays can pierce through the gas and dust in the centers of young galaxies to explore the contributions of accretion and star formation to the history of the early universe. Dark matter can be studied through the evolution of large scale structure traced by the hot X-ray emitting gas trapped in the dark matter potential.

Current models predict that the first black holes formed at redshifts of between 10 to 20 with masses of between 100,000 and 10 million solar masses. These are expected to have X-ray fluxes between $2.5 \mathrm{e}-18$ and $1.0 \mathrm{e}-17 \mathrm{erg} / \mathrm{cm} 2 / \mathrm{s}$. The goal for the XEUS sensitivity is to be able to detect these low-luminosity black holes and take detailed spectra of the high-luminosity ones. This will allow the physical properties and chemical properties of the accreting material to be studied as well as the black hole mass and possibly spin rate. The potential of these studies is shown in figure 1 where the redshifted iron line profile of a moderate mass black hole at a redshift of 10 is shown. The iron line, which has a rest energy of $6.4 \mathrm{keV}$ is clearly detected at at energy of $\sim 0.6 \mathrm{keV}$ with sufficient statistics to allow its profile to be measured in a $10^{6} \mathrm{~s}$ deep-field observation. 


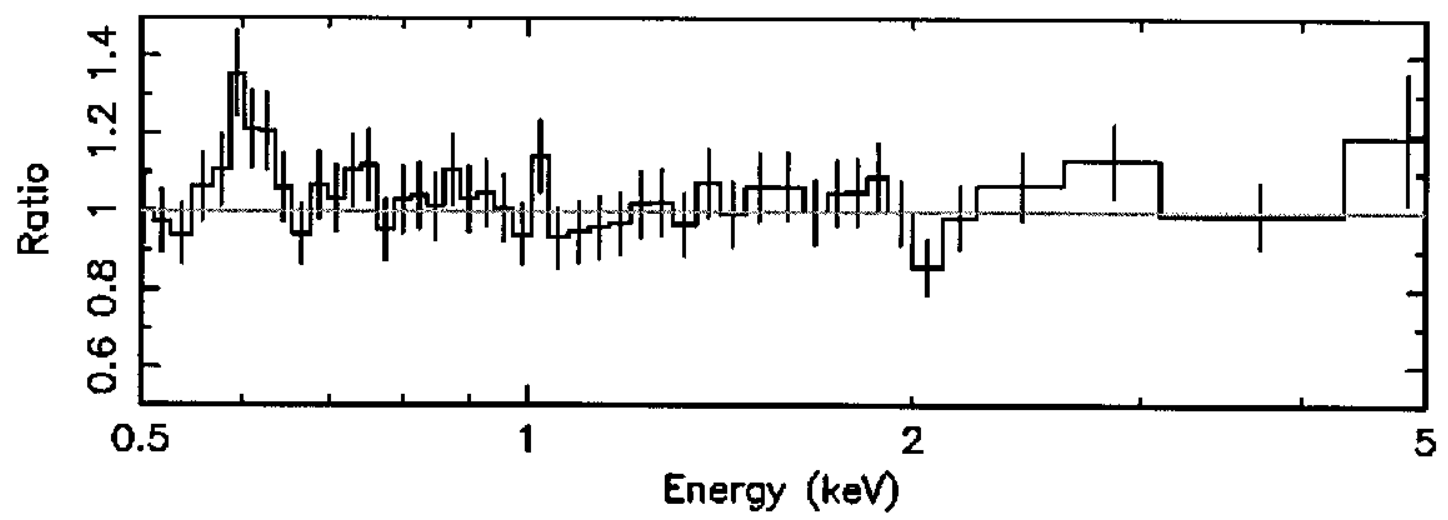

Figure1: The result of simulations of a $350 \mathrm{eV}$ equivalent width (at $\mathrm{z}=0$ ) double horned Fe line from an $\mathrm{AGN}$ at a redshift (z) of 10 with a luminosity of $10^{44} \mathrm{erg} \mathrm{s}^{-1}(2-10 \mathrm{keV}$; rest frame). An exposure of $1000 \mathrm{ks}$ using the low energy NFI is assumed together with line of sight absorption equivalent to $10^{21}$ atoms $\mathrm{cm}^{-2}$ and an intrinsic column of $5.10^{21} 1$ atoms $\mathrm{cm}^{-2}$. The Fe line is clearly detected at $0.6 \mathrm{keV}$ and its energy and profile can be measured - the key to doing astrophysics.

\section{THE XEUS BASIC DESIGN FEATURES}

The basic design goals for the XEUS Observatory are summarized below in Table I.

Table I : The Basic XEUS Design Goals

\begin{tabular}{|l|l|}
\hline Parameter & Specification \\
\hline Energy range & $0.05-30(0.1-100) \mathrm{keV}^{+}$ \\
\hline Telescope focal length & $50 \mathrm{~m}$ \\
\hline Mirror Collecting Area @ 1 keV & $30(6) \mathrm{m}^{2++}$ \\
\hline Mirror Collecting Area @ $8 \mathrm{keV}$ & $3(3) \mathrm{m}^{2++}$ \\
\hline Spatial resolution & $5 \operatorname{arcsec}(\mathrm{goal} 2 \mathrm{arcsec})$ \\
\hline Field of View & $5-10 \mathrm{arcmin}$ \\
\hline NFI energy Resolution @ 1 keV & $1 \mathrm{eV}$ \\
\hline WFI energy resolution @1 keV & $40 \mathrm{eV}$ \\
\hline Mission lifetime & $25 \mathrm{years}$ \\
\hline Orbit & LEO \\
\hline NFI = Narrow field imaging spectrometer \\
WFI = Wide field imaging spectrometer \\
'Data ( ) refer to a wider energy range under study \\
'Data ( ) refer to the initial "zero growth" configuration
\end{tabular}

The key characteristic of XEUS is the large X-ray mirror aperture combined with good angular resolution and wide-band energy response. This very demanding optics will capitalize on the successful XMM mirror technology and the industrial foundations, which have been already laid in Europe for this program. This XMM mirror technology has placed European science at the forefront in high-energy astrophysics. Unlike XMM however, where a heavily nested mirror was fabricated from closed shells, the XEUS mirror aperture of $10 \mathrm{~m}$ diameter is divided into annuli, with each annulus sub divided into sectors. The basic mirror unit therefore consists of a set of heavily stacked thin mirror plates, each retaining the correct geometry. This unit is known as a mirror petal and is a complete free standing calibrated part of the overall XEUS mirror with a spatial resolution of $\sim 5$ arcsecond (goal 2 arcsec) covering a large bandwidth from $0.05-30 \mathrm{keV}$. This is the significant technological evolution, which requires study and which should lead to a dramatic increase in capability over XMM. A schematic of a XEUS mirror petal is shown in Figure 2. 


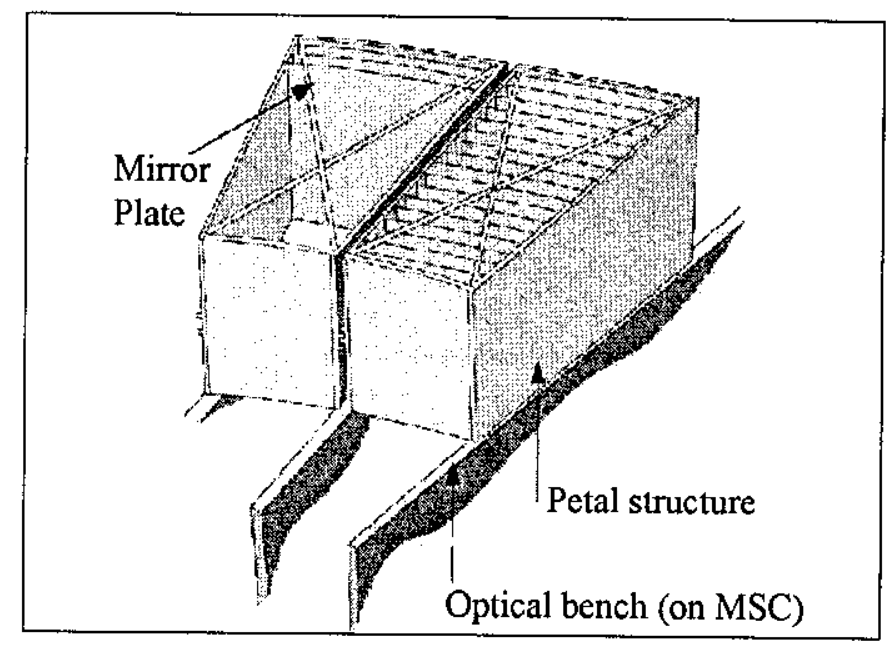

Figure 2 : A schematic of XEUS Petals

The second area of the scientific payload requiring major development will be the narrow field imaging spectrometers, which have an energy resolution of $1 \mathrm{eV}$ at $1 \mathrm{keV}$ as a goal. These instruments will have to be based on very low temperature sensors, possibly transition edge sensors or superconducting tunnel junctions operating at a temperature of 15 $300 \mathrm{mK}$.

The concept of XEUS envisages two separate spacecraft to accommodate the focal length of $50 \mathrm{~m}$, the mirror spacecraft (MSC) and the detector spacecraft (DSC).

\section{THE MIRROR SPACECRAFT (MSC1)}

The payload of MSC1 consists of the large Wolter I mirror, subdivided into mirror petals. These petals are mounted onto a stable and stiff support structure (optical bench) and are individually aligned in orbit using a dedicated optical laser system and direct observation of well established astronomical calibration fields. Each petal consists of $\sim$ 100-200 X-ray reflecting plates made by nickel electroforming on precision mandrels. All plates are integrated into the petal structure and aligned and calibrated on the ground. The petals in the MSCl (zero growth) cover a circular area with an outer and inner diameter of 4.4 and $1.3 \mathrm{~m}$ respectively. The central core is not used for petals. Provisions on the MSC1 support structure will be made to allow for the expansion of the MSC1 into MSC2. This growth provides a significant increase in the effective area at the most relevant energies for studying the high redshift universe $(\sim 1 \mathrm{keV})$. The growth is essential to push the spectroscopic limits to the highest redshifts and therefore the youngest objects.

The mirror petals are very sensitive to contamination and temperature variations. Each petal is protected from contamination during launch, maneuvering and docking with the DSCI and while in the vicinity of the ISS by hermetically sealed doors. The operating temperature of each petal must be maintained constant to $\sim 1^{\circ} \mathrm{C}$ so as to prevent deformations of the highly accurate mirror plates. The expected operating temperature of the XEUS mirror is $-35^{\circ} \mathrm{C}$. This places rather important requirements on the petal ground calibration and simulation software. While each petal contains an integrated stray light and thermal baffle as part of the unit, the MSCl will operate as a spinning spacecraft rotating about its major axis at $\sim 1$ degree/second so as to ensure the thermal requirements can be met, essentially distributing the heat uniformly around the circumference of the spacecraft. In addition the MSC contains large thermal baffles to shield the mirror from direct sunlight. The design of this baffle is critical, since the thermal loads from the sun will be periodic due to the orbit. Table II summarizes the details relating to the mirror petals and mirror. 
The $\mathrm{MSCl}$ bus is located in the central hub of the MSC1. It will be thermally isolated from the mirror optical bench and maintained at a constant $20^{\circ} \mathrm{C}$. This bus provides power and telemetry (to ground and DSC1), controls the attitude, thermal environment and docking. The MSC1 telemetry contains only housekeeping data, involving only low data rates.

\section{Table II : Details of the XEUS Mirror}

\begin{tabular}{|l|l|}
\hline \multicolumn{1}{|c|}{ Parameter } & \multicolumn{1}{c|}{ Specification } \\
\hline Mirror plate material & Nickel \\
\hline X-ray reflecting surface & Gold \\
\hline Plate thickness & 300 microns \\
\hline Plate length and width (typical) & lm x 0.5 m \\
\hline Number annuli (zero growth) & 2 \\
\hline Number of petals (zero growth) & $2 \times 16$ \\
\hline Petal Baffles (entry \& exit) & $2 \times 100 \mathrm{~mm}$ \\
\hline Typical Petal Mass & $200-350 \mathrm{~kg}$ \\
\hline Mirror mass (zero growth) & $8900 \mathrm{~kg}$ \\
\hline Number of annuli added & 3 \\
\hline Number of sectors added & 8 \\
\hline Number of petals/sector & 12 \\
\hline Diameter of full grown mirror & $10 \mathrm{~m}$ \\
\hline Mass of mirror fully grown & $17400 \mathrm{~kg}$ \\
\hline
\end{tabular}

The MSC will be flying in low earth orbit but will not actively control its orbit during observations. The MSCl will have a complete attituse and orbit control system, compatible with the requirements for docking to the ISS. Major orbit changes, e.g. for visiting the ISS, will be performed using the DSC1 orbit control system after docking to the DSC1. The pointing direction of the $\mathrm{MSCl}$ will be restricted due to stray light, thermal and power requirements. The angle between the telescope pointing and the sun-vector will always be in the range $90-120^{\circ}$ during the observation phase of the mission. Slews between individual XEUS fields will be short $\left(\leq 10^{\circ}\right)$ and will be executed during the daylight part of the orbit. Any repointing of XEUS will require a coordinated sequence of activities involving the MSCl and the DSCI attitudes and the DSC1 position relative to the MSC1. Typical expose times per pointing are envisaged to be $10^{5}$ to $10^{6}$ seconds. Note the pointing accuracy of the MSCl does not need to be particularly high ( goal of 10") due to the field of view of the XEUS mirror (5-10'). The actual attitude will be measured to a much higher accuracy with a-posteriori reconstruction of $\leq 1$ ". Finally the MSC1 will provide references to the DSC1 to establish the relative position of the two spacecraft via a laser alignment system on the main axis of symmetry.

The docking port is located on the mirror entry plane and supported on a rotating platform, simplifying the robotic addition of mirror sectors at the ISS. Figures 3 and 4 show the MSC1 in side view and cross section. 


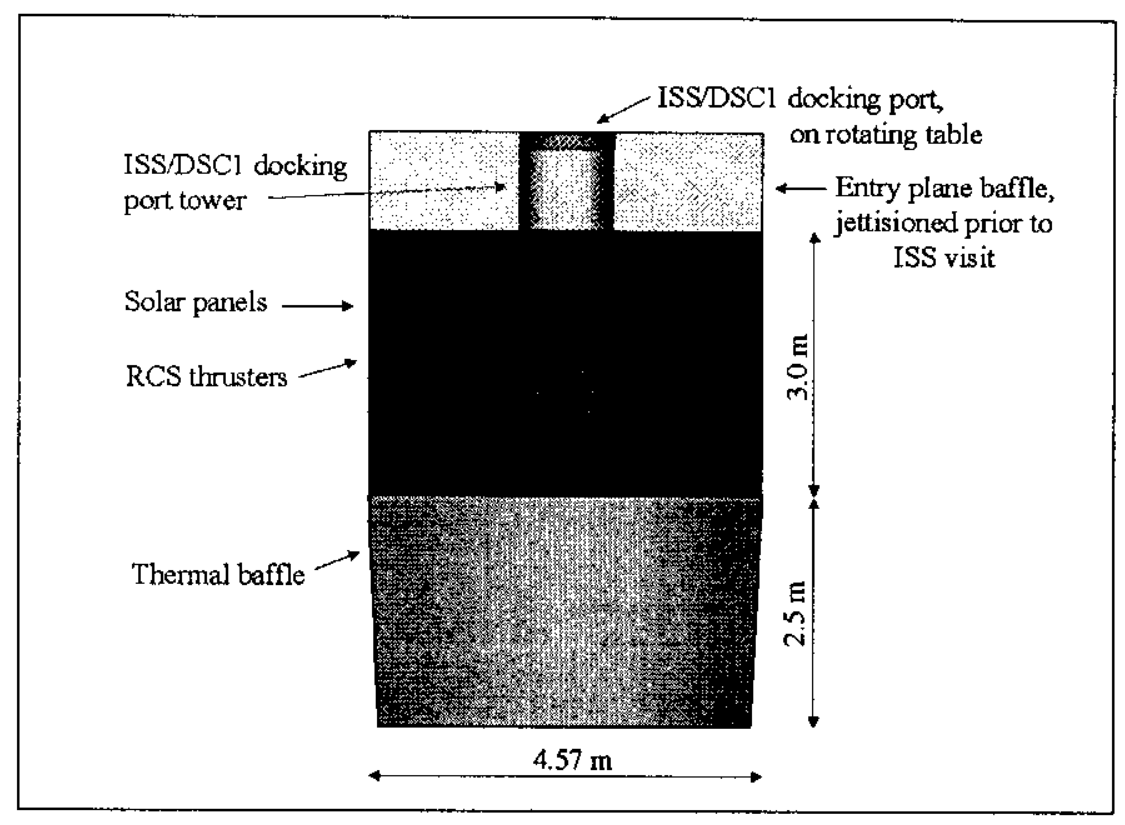

Figure 3: MSC1, side view

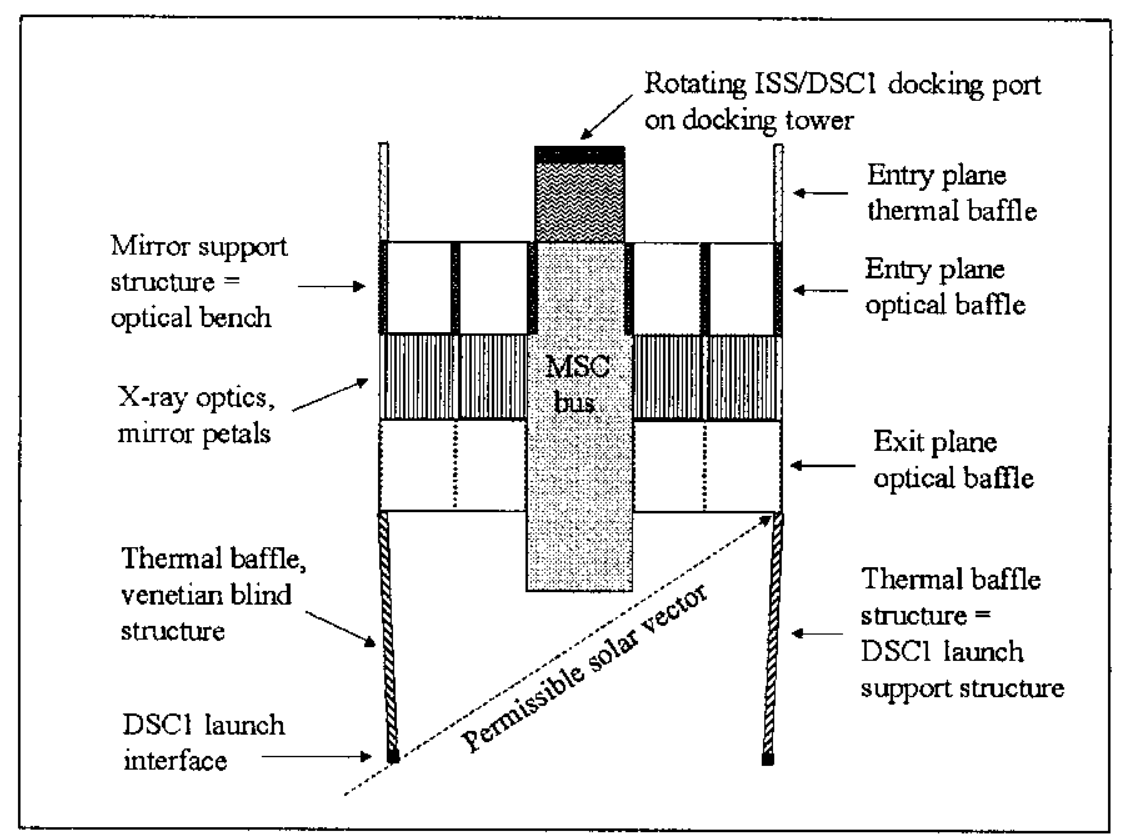

Figure 4: MSC1, cross section

\section{THE DETECTOR SPACECRAFT (DSC1)}

The X-ray detectors (spectrometers and imager) are the payload of the DSC. The DSC1 will be tracking the focus of the Xray telescope (MSCl) to within $\pm 2 \mathrm{~mm}$. This implies that the DSC1 will be flying in a non-Keplerian orbit. The orbital characteristics of this MSCI-DSCl tandem pair is summarized in Table III.

This table refers to the nominal mission start with XEUS inserted directly into the Fellow Traveler Orbit (FTO). This orbit will naturally decay with a mean and maximum orbit decay rate of -0.55 and $-4.7 \mathrm{~km} /$ year respectively. The $\mathrm{DSCl}$ will be 
flying in a similar orbit, but simply shifted by $50 \mathrm{~m}$ against the pointing direction of XEUS. The peak forces required to keep the DSCl on station in this orbit depend on the angle $\alpha$ between the XEUS orbital plane and the XEUS pointing direction. The smallest force is required for the largest $\alpha$. For $0<\alpha<90^{\circ}$ the average force is $540-350 \mathrm{mN}$ requiring a dv of $\sim 0.3-$ $0.5 \mathrm{~m} / \mathrm{s}$ per orbit. Thus observations at high declinations will require less propellant and will therefore be preferred in the mission planning. All other forces acting on the DSCl are about two orders of magnitude lower than the non-Keplerian orbit forces and can be compensated by the DSCl orbital control system. Table IV summarizes the requirements with respect to the $\mathrm{DSCl}$ orbit control system based on the average of the forces discussed above.

\section{Table III :Orbital Characteristics of the DSC/MSC}

\begin{tabular}{|l|l|}
\hline \multicolumn{1}{|c|}{ Parameter } & \multicolumn{1}{c|}{ Specification } \\
\hline Altitude & $600 \mathrm{~km}$ \\
\hline Eccentricity & 0 \\
\hline Inclination & $51.6 \mathrm{deg}$ \\
\hline Period & $97 \mathrm{~min}$ \\
\hline Maximum eclipse & $35.5 \mathrm{~min}$ \\
\hline Node spacing & $24 \mathrm{deg}$ \\
\hline Node precession & $-4.5 \mathrm{deg} / \mathrm{day}$ \\
\hline De-orbit dv & $256 \mathrm{~m} / \mathrm{s}$ \\
\hline Altitude change dv & $0.54 \mathrm{~m} / \mathrm{s} / \mathrm{km}$ \\
\hline Plane change dv & $132 \mathrm{~m} / \mathrm{s} / \mathrm{deg}$ \\
\hline Earth angular radius & $66 \mathrm{deg}$ \\
\hline Range to horizon & $2831 \mathrm{~km}$ \\
\hline
\end{tabular}

Table IV : Details of the DSC Orbit Control

\begin{tabular}{|l|l|}
\hline \multicolumn{1}{|c|}{ Parameter } & \multicolumn{1}{c|}{ Specification } \\
\hline DSC1 launch mass $(\mathrm{kg})$ & 6000 \\
\hline Peak Forces (worst case) & $700 \mathrm{mN}$ \\
\hline Average dv over 1 orbit & $0.37 \mathrm{~m} / \mathrm{s}$ \\
\hline Average dv over 1 day & $5.6 \mathrm{~m} / \mathrm{s}$ \\
\hline DSC FTO to ISS dv & $111 \mathrm{~m} / \mathrm{s}$ \\
\hline
\end{tabular}

Static plasma thrusters (SPT) will provide the force to keep the DSC1 in it's orbit. These thrusters must be distributed such, that the thrust axis distribution is as isotropic as possible in order to minimize thruster power and propellant. This need arises from the orbital inclination and its precession coupled to the different pointing directions required by XEUS., which leads to constantly changing forces around the orbit. Issues related to the lifetime, cycle times and thrust control and dynamic range will be important for the final thruster selection.

The DSCl dry mass is $\sim 3100 \mathrm{~kg}$. About $2900 \mathrm{~kg}$ of inert Xenon propellant will be loaded into the DSC1 before launch, giving the DSC1 an observation lifetime of about 5 years. The DSC1 will require about $14 \mathrm{~kW}$ of power for the worst case eclipse, of which $13 \mathrm{~kW}$ are for the electric propulsion system.

Figure 5 shows a perspective view of DSC1 looking at the anti-solar side, which shows the thermal radiators of the instruments. Solar panels are sized for the DSCl power requirements and are shown in figure 5 positioned for the case when XEUS is pointing at a low declination target, i.e. the array normal is tilted $120^{\circ}$ from the XEUS optical axis. Note that the solar array is always kept perpendicular to the sun. The DSCl is $\sim 2.8 \mathrm{~m}$ cube with deployable solar arrays that have a full span of $26 \mathrm{~m}$ and a width of $\sim 7.9 \mathrm{~m}$. 


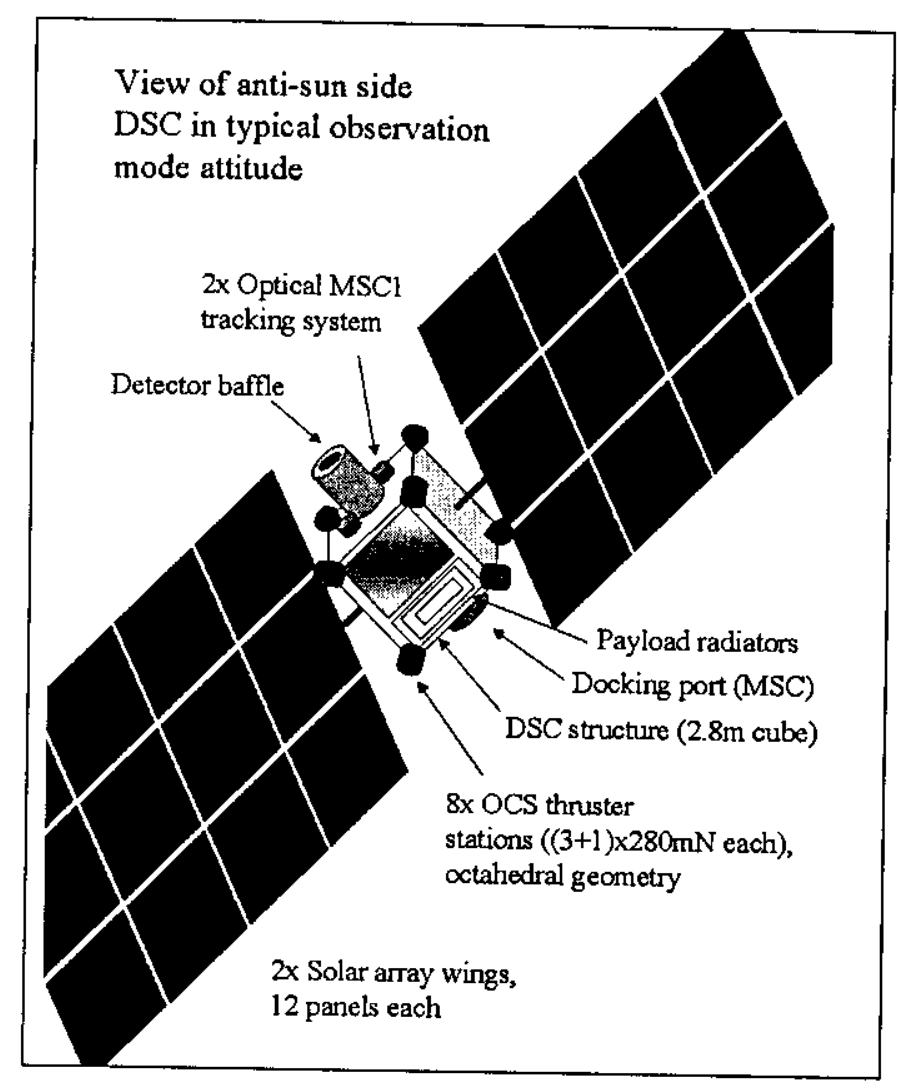

Figure 5: DSC1, perspective view

The payload of DSC1 will include three X-ray imaging spectrometers (WFI, NFI1 and NFI2). Their main characteristics are summarized in Table V. The payload is subject to a on-going detailed study and as such the data in table V should be considered as provisional.

Table V : The DSC1 Payload Characteristics

\begin{tabular}{|l|l|l|}
\hline \multicolumn{1}{|c|}{ Characteristic } & \multicolumn{1}{|c|}{ WFI } & \multicolumn{1}{c|}{ NFI1/2 } \\
\hline Detector type & CCD & TES \& STJ \\
\hline Operating Temp. & $200-260 \mathrm{~K}$ & $15-300 \mathrm{mK}$ \\
\hline Cooling & Radiator & Stirling+ADR \\
\hline Detector Size & $70 \times 70 \mathrm{~mm}^{2}$ & $7 \times 7 \mathrm{~mm}^{2}$ \\
\hline Pixel size & $40 \mu \mathrm{m}$ & $100 \mu \mathrm{m}$ \\
\hline Time Resolution & $5 \mu \mathrm{s}(1 \mathrm{~ms})$ & $1-5 \mu \mathrm{s}$ \\
\hline Energy range & $0.1-30 \mathrm{keV}$ & $0.05-3$ (NFI1) \\
\hline & & $0.5-10(\mathrm{NFI} 2)$ \\
\hline Mass (total) & $60 \mathrm{~kg}$ & $350 \mathrm{~kg}$ \\
\hline Power (total) & $60 \mathrm{~W}$ & $300 \mathrm{~W}$ \\
\hline
\end{tabular}

Note that the WFI can be cooled passively using a multiple stage radiator on the anti-sun side of DSCl. The effective environmental heat sink temperature in the XEUS orbit will not be lower than $190 \mathrm{~K}$ for some selected DSCl locations avoiding the sun exposure. For the two narrow field imaging spectrometers flat panel radiators with heat pipes will dissipate the thermal energy from the closed cycle mechanical coolers that are used as the main cooling stage to a base temperature of $2 \mathrm{~K}$. The cooling to $300 \mathrm{mk}$ or $15 \mathrm{mK}$ will be achieved using a closed cycle ${ }^{3} \mathrm{He}$ sorption pump or an ADR respectively. 


\section{THE XEUS MISSION PROFILE}

The XEUS spacecraft consists of two free flying spacecraft: a detector spacecraft (DSC) and a mirror spacecraft (MSC) separated by $\sim 50 \mathrm{~m}$ and aligned in the low earth orbit by an active orbital control and alignment system. Such a large aperture mirror cannot be deployed in a single launch. In the current baseline scenario it is envisaged to launch the "zero growth" XEUS mated pair (MSC1+DSC1) directly into a Fellow Traveler Orbit (FTO) to the ISS using an Ariane 5e or similar launcher. The FTO is a low earth orbit, altitude $\sim 600 \mathrm{~km}$ with an inclination similar to the ISS. The mated pair will decouple in FTO and the DSCl will take up station $50 \mathrm{~m}$ from the MSCl. After alignment validation and normal spacecraft/payload checkout the "zero growth" astrophysics observation program will commence. The MSC will point at a given target field and maintain a stable attitude while the DSC1 will maintain the focal distance and alignment with respect to the MSCl so that the field image as measured by the DSCl detectors remains stable.

It must be stressed that XEUS is in this scenario completely autonomous from the ISS. In the initial launch "zero growth" configuration the MSCl will contain only the two inner annuli of the telescope filled with 32 petals. The XEUS initial collecting area is however huge $\left(\sim 6 \mathrm{~m}^{2}\right.$ at $\left.1 \mathrm{keV}\right)$ but still has substantial room for growth through use of the ISS. This growth capability is crucial for achieving the ultimate scientific aims of the mission.

The pair of XEUS spacecraft can however be re-mated in orbit and, through the use of the orbit control system (OCS) on DSC1, allow the mated pair to perform an orbit change and come to the vicinity of the ISS. At this point the pair is able to wait for up to one year by following the ISS in a safe distance, until the later is ready to receive the MSC1 for refurbishment and growth. Prior to the docking to the ISS the DSC1 will separate from the MSC1, and then the MSC1 will approach and dock to the ISS. As far as possible, docking technology developed for the Automated Transfer Vehicle (ATV) will be used. The DSCl, after de-docking from the MSCl and it's usefulness at an end, will undergo a controlled de-orbit.

It must be stressed, that XEUS will be able to start its astrophysics observing program effectively from day 1 after launch of the "zero growth" configuration into FTO.

\section{THE ACTIVITIES AT THE ISS}

In the current baseline scenario it is envisaged that the mated XEUS pair (MSC1 +DSC1) will arrive in the vicinity of the station from FTO and the MSCl will then dock at the ISS using the same docking port as an ATV on the Russian segment. This rendezvous with the ISS, which will take advantage of the evolution of the XEUS orbit with respect to the ISS, will occur on a timescale of $\sim 4-5$ years. At the ISS the MSC1 is grown to MSC2.

In preparation for the $\mathrm{MSCl}$ visit to ISS the required 8 mirror sectors, each containing 12 petals, will be brought to the ISS. This may occur close to or at the time of the MSC1 visit. A Space Shuttle could transport the required 8 sectors in one flight (see figure 6). Alternatively a period of up to 5 years is available for individual transport of sectors to the ISS, in which case external storage of the mirror sectors is required. The mirror sector structure serves as the transport container.

The top level activity envisaged at the ISS after the MSC1 has docked with the ISS can be summarized as follows :

- Retrieve the sectors required to expand the mirror from the zero growth $\sim 4.5 \mathrm{~m}$ diameter to the final diameter of $\sim 10 \mathrm{~m}$ from external storage areas or the transport vehicle.

- Insert the 8 sectors into MSCl mirror support structure. Note that each mirror sector is $\sim 1600 \mathrm{~kg}$ (see figure 7)

- Deploy the thermal baffle elements of the MSC2 (figure 8).

- Perform checkout of MSC2.

- De-dock MSC2 and move it to the ISS safety perimeter.

- Transfer MSC2 orbit to FTO.

After MSC2 arrives in FTO, DSC2 is launched into the MSC2 orbit and the fully grown Xeus starts observations. 


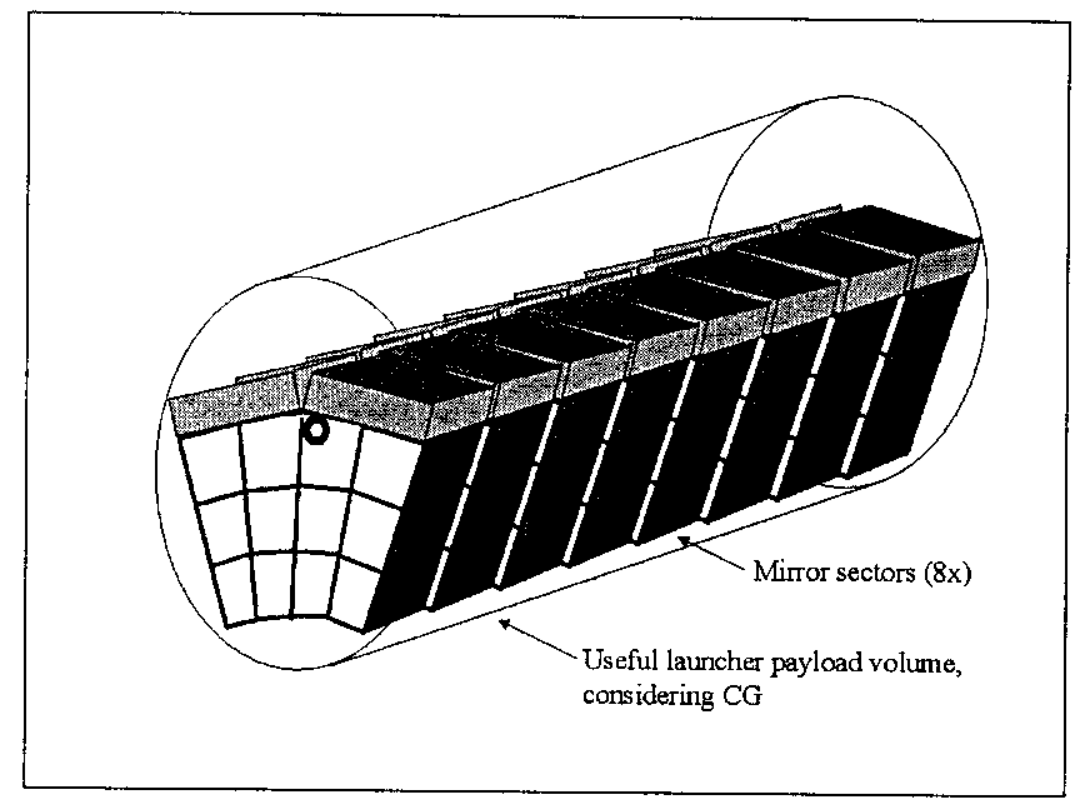

Figure 6: Stowage of mirror sectors and thermal baffle elements (CG: center of gravity)

The mirror growth phase at the ISS may involve the use of robotics such as the European Robotics Arm for handling petals and a limited amount of EVA. The SSRMS might be used to transport the sectors from the storage area or the transport vehicle to the MSC1. The mirror sectors will contain jigs and tool points for robotic manipulation. For cleanliness reasons the mirror sectors are hermetically sealed.

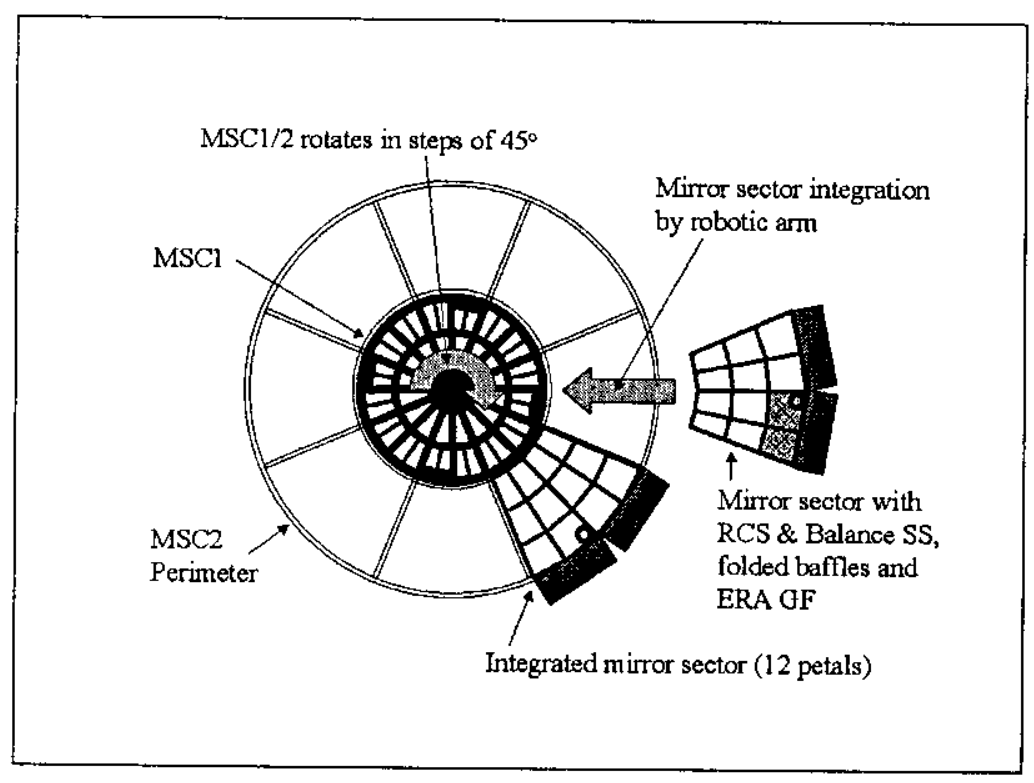

Figure 7: A schematic of the XEUS mirror growth 


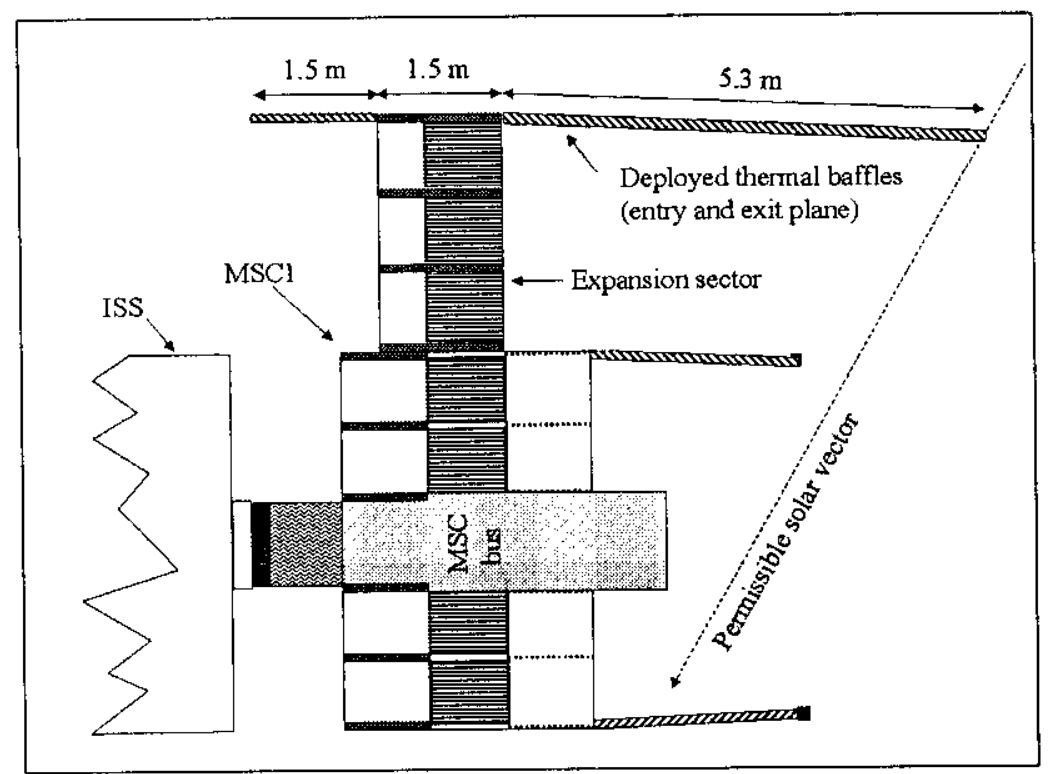

Figure 8: Cross section of MSC docked to the ISS

Key issues regarding the ISS activities, which need addressing, are :

- The logistics and costs involved in the transport and stowage of sectors and baffle elements at the ISS.

- The external storage locations and capability at the ISS.

- Technical issues relating to the transport of sectors at the ISS

- Sector insertion into the MSC1 support structure using ERA.

- Docking of MSC1 to the ISS and de-docking of MSC2 from the ISS.

- Possibility of berthing XEUS (MSC2 and/or DSC2) near to the ISS for repairs or consumable replenishment.

This baseline strategy allows Xeus to take advantage of the key attributes of the ISS as an in-orbit assembly facility, while minimizing the complexity of the tasks and resource demands required of the ISS. In addition the delay between the zero growth launch into FTO and the rendezvous with the ISS allows :

- The costs of production of the mirror to be spread over a period of more than 10 years.

- The continual improvement in the mirror petal technology with time.

- The spreading of the launch costs.

- The production of a series of low cost DSC busses spread over 10 years or more. Note that at spacecraft level all busses after DSCl are identical since the MSC1 has evolved into the final mirror MSC2.

- Development of new improved focal plane instrumentation incorporated into DSC2 and follow on DSCs.

Future DSCs after DSC2 are envisaged, but these can, like DSC2, be launched directly into FTO, with new instruments and consumables. The MSC2 contains a fully expanded mirror system at this stage. Future access to the MSC2, either for refurbishment or replenishment of consumables, can be either performed directly by the Space Shuttle or possibly through a re-docking to ISS or berthing near to the ISS. 


\section{CONCLUSIONS}

The XEUS mission represents an ambitious project full of new approaches and technologies. At payload level the most demanding issues seriously needing attention over the next few years are:

- The development and demonstration of the $2-5$ arc second performance of the basic mirror petal unit

- The development of efficient low temperature imaging spectrometers providing a resolution below $1 \mathrm{eV}$ and $<10 \mathrm{eV}$ at 1 and $8 \mathrm{keV}$ respectively.

- The development of a large format high- speed wide field imaging solid state spectrometer.

- The development of the low temperature closed cycle cooling system.

At the spacecraft and mission level a number of technical and logistical issues need to be addressed as follows :

- The DSCl orbit control system and the optimization of the propulsion system.

- The DSC1-MSCl metrology system

- The transport to the ISS and possibly storage of a large amount of cargo externally at the ISS

- The rendezvous and docking of MSCl to the ISS

- The feasibility of the robotics and EVA activity while MSC1 is docked at the ISS

\section{ACKNOWLEDGEMENTS}

Many people have been involved in the development of the ideas for the XEUS project over the last $2-3$ years. This has culminated in the mission profile described in this paper. The level of technical detail presented here would not have been possible without the enthusiastic support of the ESA XMM project team led by R. Laine. Particular thanks however to team members: T. van der Laan (design and mechanical issues), D. Stramaccioni (thermal), K. van Katwijk (system engineering with respect to the mirror), D. de Chambure (XEUS mirror petal). Very important contributions on the electric propulsion were given by J. del Amo Gonzalez, ESA TOS. Finally A. Linssen is thanked for helping us get the XEUS mirror petal development program underway. 


\section{The X-ray Evolving Universe Spectroscopy Mission (XEUS) The X-ray mirror design and technology}

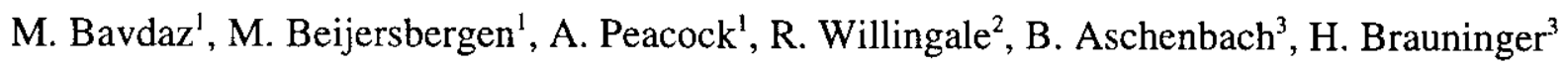

'Space Science Department of ESA, ESTEC, Noordwijk, The Netherlands ${ }^{2}$ Department of Physics and Astronomy, Leicester University, United Kingdom ${ }^{3}$ Max Planck Institut für Physik und Astrophysik, Garching,

ESLAB 1998/052/SA

To be published in SPIE Proceedings V3766

Presented at the SPIE International Symposium on Optical Science, Engineering and Instrumentation, 18-23 July 1999, Denver, USA 